\title{
Acute gastrointestinal haemorrhage in patients treated with anticoagulant drugs
}

The reported incidence of haemorrhagic complications in patients treated with anticoagulated drugs ranges from 12 to $40 \%{ }^{1-4}$ The first year of anticoagulation carries the highest risk of bleeding. A recent meta-analysis concluded that during this period $5 \%$ of bleeding episodes will be life threatening and $1 \%$ of patients will die from uncontrolled haemorrhage. ${ }^{5}$ Subsequently the annual bleeding rate is reduced by about $50 \%$. The commonest site of significant bleeding is the gastrointestinal tract and this leading article considers the problems of acute, life threatening gastrointestinal bleeding in patients taking oral anticoagulant drugs.

Several reviews have identified factors that influence the source and severity of gastrointestinal bleeding in patients taking anticoagulants. The risk is greatest in those with the most prolonged prothrombin time $e^{6-10}$; it is increased by concomitant aspirin consumption. ${ }^{11} 12$ Increasing age, previous gastrointestinal bleeding, atrial fibrillation, and serious coexisting conditions such as renal insufficiency and anaemia are other adverse prognostic factors, 35910 while the nature of the bleeding site is an obvious important factor.

\section{Who and how to investigate?}

Gastrointestinal bleeding that occurs when the international normalised ratio (INR) is less than 3 almost invariably originates from a discrete mucosal lesion ${ }^{9}{ }^{13-15}$; peptic ulcer is the commonest cause. Appropriate endoscopic and radiological investigations will usually identify the bleeding point. Investigations for gastrointestinal bleeding that occur when the patient is overanticoagulated (INR greater than 6) tend to be less rewarding, but it is our view that virtually all patients who develop bleeding while receiving anticoagulants merit investigation, irrespective of their admission INR as many will have mucosal lesions that are amenable to endoscopic diagnosis and treatment. Conservative treatment alone leaves these patients at risk of continued bleeding and simply attributing gastrointestinal bleeding to overanticoagulation alone is dangerous. The downside of this approach is that many patients are treated with anticoagulants for severe cardiovascular disease, which increases the hazards of endoscopy. Gastroduodenoscopy and colonoscopy should not be undertaken lightly in these patients, who may be bleeding acutely and who often have significant cardiovascular disease but should be done by experts with the ability to undertake a range of endoscopic treatments and who have the backup of appropriate monitoring, cardiovascular support, and expert nursing assistance.

About half of the patients who bleed from the gastrointestinal tract while taking oral anticoagulants do so from the stomach or duodenum, usually from a peptic ulcer. ${ }^{15-17}$ About a quarter bleed from the colon, commonly from polyps, diverticular disease, vascular malformations or cancer. A few bleed from the small bowel (leiomyoma or Meckel's diverticulum) and the rest are undiagnosed. Clinical presentation can be a poor guide to the site and cause of haemorrhage; bright red rectal bleeding may be due to bleeding duodenal ulcer, colonic bleeding can present as 'melaena'. It follows that some patients will require investigation of both the upper and lower gastrointestinal tract before the clinician can be confident of the bleeding source. After resuscitation this is best done endoscopically during a single session. Unless bleeding obviously originates from the anal canal, upper endoscopy is done first and may show active bleeding or recent haemorrhage from a major lesion. If gastroscopy is negative, the endoscopist should be prepared to immediately undertake colonoscopy. Colonoscopy is obviously technically demanding in this context and may need to be repeated after colonic lavage. Patients who undergo negative upper and lower endoscopy and who continue to bleed are considered for visceral angiography (when bleeding is brisk and expertise is available). Enteroscopy is an option for more modest bleeding.

\section{When to reverse anticoagulation?}

Patients are usually treated with anticoagulants for good reason and the clinician faced with the patient bleeding from the gastrointestinal tract is confronted with the difficult decision of reversing anticoagulation and risking thromboembolic consequences or continuing warfarin and risking exsanguination. Some indications for anticoagulation are more critical than others. Hence the hazards of reversing anticoagulation in a patient receiving anticoagulants for lone atrial fibrillation, transient ischaemic episodes or dilated cardiomyopathy in which the therapeutic INR is $2-3$ are less than those for patients treated for prosthetic metal heart valves or frequent pulmonary emboli in whom an INR of 3-4.5 is recommended. For the patient who presents with life threatening bleeding the risks of thrombosis have to be accepted and anticoagulation reversed, not because of the potential hazards of endoscopy (which are probably only slightly increased in patients receiving anticoagulants) but because of the probability that bleeding will continue unless this is done. Furthermore, endoscopic treatment is probably safest done after reversal of anticoagulation and it therefore seems wise to recommend this before endoscopy.

Ideally, controlled reversal of anticoagulation is achieved using prothrombin complex concentration. ${ }^{18}$ This is not freely used for gastrointestinal haemorrhage but perhaps should be considered in the most critical patients who are bleeding briskly. In practice fresh frozen plasma is used to replace deficient clotting factors but this may not be as effective. Vitamin $\mathrm{K} 1$ given by itself is not suitable for active bleeding because it acts slowly. The British National Formulary recommends that vitamin $\mathrm{K} 1(5 \mathrm{mg})$ is infused with fresh frozen plasma to correct clotting in patients receiving anticoagulants who are bleeding. Whether this is 
always correct is a matter of debate; vitamin $\mathrm{K} 1$ infusion makes subsequent re-anticoagulation difficult. The extent to which anticoagulation is reversed is not clear; the INR that the clinician aims to achieve after fresh frozen plasma is a compromise between the (unknown) risks of further bleeding and the (unknown) risks of thrombosis in that patient.

\section{Treatment options}

For the patient not receiving anticoagulant treatment, endoscopic investigation has become the treatment of choice for a range of bleeding lesions throughout the gastrointestinal tract and surgery is now reserved for the failures of therapeutic endoscopy. ${ }^{19}$ Until recently the safety and efficacy of these techniques has not been assessed in patients taking anticoagulants but our own experience suggests that, at least for the patient presenting with peptic ulcer haemorrhage, the same is true for patients receiving anticoagulants, as for those not receiving warfarin. ${ }^{17} \mathrm{We}$ reviewed the clinical course of 23 patients who presented with major peptic ulcer haemorrhage while taking warfarin and compared their outcome with that of a closely matched group of patients who presented with ulcer bleeding in the absence of anticoagulant treatment. The groups were well matched in respect of age, admission haemoglobin concentration, the presence of shock, and endoscopic findings. The INR of the warfarin group was partially corrected using fresh frozen plasma to $1 \cdot 5-2 \cdot 5$ and endoscopic treatment (injection or the heater probe) was attempted by an experienced endoscopist in all patients. The incidence of uncontrolled bleeding ( 17 and $18 \%$ ), need for emergency surgery ( 9 and $8 \%$ ) were similar in both groups; the study was too small to make sensible comments about mortality. No significant complications developed in any patient and it seems reasonable, albeit on the basis of this small study, to suggest that patients receiving anticoagulants should be treated endoscopically for bleeding ulcer disease.

The efficacy of endoscopic treatment in patients taking anticoagulants bleeding from the colon has not been critically assessed. It is likely that colonic polypectomy for a bleeding adenoma will be safe and effective, but what of bleeding vascular malformations or diverticular disease? We do not know whether Nd-Yag photocoagulation of colonic vascular malformations is worthwhile; indeed this approach is often unsuccessful in patients not receiving anticoagulants. ${ }^{20}$ Alternative treatments such as oestrogen therapy, right hemicolectomy or even aortic valve replacement are all hazardous options and treatment is unsatisfactory for the patient who needs longterm anticoagulant treatment.

\section{Longterm outcome}

The chances of rebleeding after successful haemostatic treatment depend on the underlying cause of bleeding. Our experience suggests that anticoagulation can be safely restarted a few days after haemostasis of bleeding ulcers has been achieved; no further episodes of haemorrhage occurred in our own group of patients receiving anticoagulants over a median follow up period of eight months. Lifelong ulcer healing treatment seems a sensible option in this group.

The outcome for other patients is unclear. A life threatening bleed from vascular malformations is probably a contraindication to re-anticoagulation although decisions have to be based upon relative risks in any single patient. The group of patients who undergo extensive negative investigations for serious haemorrhage are a particularly difficult group. The presumption is often that bleeding has occurred from vascular malformations somewhere in the gut. Again decisions are based upon the severity of bleeding balanced against the indication for anticoagulation. Our own experience suggests that these patients can usually safely be given anticoagulants again with low risk of further major haemorrhage.

\section{Conclusions}

All patients who are treated with anticoagulants and present with major gastrointestinal haemorrhage merit endoscopic diagnosis and treatment. Anticoagulation should be reversed in a controlled manner using clotting factors and trying to give some protection against thrombosis while not making subsequent reanticoagulation difficult. Careful consideration is necessary before anticoagulation is restarted after bleeding has been controlled and a less intense anticoagulant regimen considered to balance the risks of rebleeding against those of thromboembolism.

C P CHOUDARI K R PALMER

Gastrointestinal Unit,

Western General Hospital,

Edinburgh EH4 $2 \mathrm{XU}$

1 Coon WW, Willis PW III. Haemorrhagic complications of anticoagulant therapy. Arch Intern Med 1974; 133: 386-92.

2 Forfar JC. A 7-year analysis of haemorrhage in patients on long-term anticoagulant treatment. Br Heart f 1979; 42: 128-32.

3 Levine MN, Raskob G, Hirsh J. Haemorrhagic complications of long-term anticoagulant therapy. Chest 1989; 95: 26-36S.

4 Landefeld CS, Cook EF, Flatley M, Weisberg M, Goldman L. Identification and preliminary validation of predictors of major bleeding in hospitalized and preliminary validation of predictors of major bleeding in hospitaliz

patients starting anticoagulant therapy. $A m \mathcal{F}$ Med 1987; 82: 703-13.
5 Landefeld CS, Beyth RJ. Anticoagulant-related bleeding: clinical epidemiology, prediction, and prevention. Am f Med 1993; 95: 315-28.

6 Hull R, Hirsh J, Jay R, Carter C, England C, Gent M, et al. Different intensities of oral anticoagulant therapy in the treatment of proximal-vein thrombosis. $N$ Engl $\mathcal{F}$ Med 1982; 307: 1676-81.

7 Turpie AGG, Gunstensen J, Hirsh J, Nelson H, Gent M. Randomised comparison of two intensities of oral anticoagulant therapy after tissue heart valve replacement. Lancet 1988; i: 1242-5.

8 Saour JN, Sieck JO, Mamo AR, Gallus AS. Trial of different intensities of anticoagulation in patients with prosthetic heart valves. $N$ Engl $\mathcal{F}$ Med 1990; 322: 428-32.

9 Landefeld CS, Rosenblatt MV, Goldman L. Bleeding in out-patients treated with warfarin: relation to the prothrombin time and important remediable lesions. Am f Med 1989; 87: 153-9.

10 Landefeld CS, Goldman L. Major bleeding in out-patients treated with warfarin: incidence and prediction by factors known at start of outpatient therapy. $A m \mathcal{F} M e d$ 1989; 87: 144-52.

11 Dale J, Myhre E, Loew D. Bleeding during acetylsalicylic acid and anticoagulant therapy in patients with reduced platelet reactivity after aortic valve replacement. Am Heart f 1980; 99: 746-52.

12 Chesebro JH, Fuster V, Elveback LR, McGoon DC, Pluth JR, Puga FJ, et al. Trial of combined warfarin plus dipyridamole or aspirin therapy in prosthetic heart valve replacement: danger of aspirin compared with dypiridamole. Am $\mathcal{F}$ Cardiol 1983; 51: 1537-41.

13 Assessment of short-term anticoagulant administration after cardiac infarction: report of the working party on anticoagulant therapy in coronary thrombosis to the Medical Research Council. BMf 1969; 1: 335-42.

14 Zweifler AJ. Relation of prothrombin concentration to bleeding during oral anti-coagulant therapy. Its importance in detection of latent organic anti-coagulant therapy. Its importance
lesions. $N$ Engl $f$ Med 1962; 267: 283-5.

15 Wilcox CM, Truss CD. Gastrointestinal bleeding in patients receiving longterm anticoagulant therapy. Am $\mathcal{A}$ Med $1988 ; 84: 683-90$.

16 Tabibian N. Acute gastrointestinal bleeding in anticoagulated patients: a prospective evaluation. Am $\mathcal{f}$ Gastroenterol 1989; 84: 10-2.

17 Choudari CP, Palmer KR. Acute gastrointestinal haemorrhage in anticoagulated patients: diagnoses and response to endoscopic treatment. Gut 1994; 35: 464-6.

18 British Society of Haematology. Guidelines on oral anticoagulation. $\mathcal{f}$ Clin Pathol 1990; 43: 177-83.

19 Cook DJ, Guyatt GH, Salena BJ, Laine LA. Endoscopic therapy for acute nonvariceal upper gastrointestinal haemorrhage: a meta-analysis. Gastroenterology 1992; 102: 139-48.

20 Machicado GA, Jensen DM. Upper gastrointestinal angiomata. Gastroenterol Clin North Am ????; 1: 241-63. 